\title{
Safe SUSY
}

\section{Borut Bajc, ${ }^{a}$ Nicola Andrea Dondi ${ }^{b}$ and Francesco Sannino ${ }^{b}$}

\author{
${ }^{a}$ J. Stefan Institute, \\ 1000 Ljubljana, Slovenia \\ ${ }^{b} C P^{3}$-Origins 8 the Danish IAS, University of Southern Denmark, \\ Campusvej 55, Odense $M$ 5230, Denmark \\ E-mail: borut.bajc@ijs.si, dondi@cp3.sdu.dk, sannino@cp3.sdu.dk
}

\begin{abstract}
We investigate the short distance fate of distinct classes of not asymptotically free supersymmetric gauge theories. Examples include super QCD with two adjoint fields and generalised superpotentials, gauge theories without superpotentials and with two types of matter representation and semi-simple gauge theories such as quivers. We show that for the aforementioned theories asymptotic safety is nonperturbatively compatible with all known constraints.
\end{abstract}

KEYWORDs: Supersymmetric Gauge Theory, Supersymmetry and Duality

ARXIV EPRINT: 1709.07436 


\section{Contents}

1 Introduction 1

2 Safe SQCD with two adjoints and superpotential 2

3 Safety without superpotentials: the $\mathrm{SO}(10)$ and $\mathrm{SU}(5)$ templates 4

3.1 The $\mathrm{SO}(10)$ template 4

3.2 The SU(5) template 6

$\begin{array}{lll}4 & \text { Semi-simple gauge groups } & 7\end{array}$

$\begin{array}{lll}4.1 & \text { The } \mathrm{SU}(\mathrm{N})^{4} \text { quiver } & 7\end{array}$

4.2 An $\mathrm{SU}\left(\mathrm{N}_{1}\right) \otimes \mathrm{SU}\left(\mathrm{N}_{2}\right)$ example $\quad 9$

5 Conclusions $\quad 11$

\section{Introduction}

The discovery of asymptotic freedom [1,2] has played an important role in particle physics. According to Wilson $[3,4]$ these theories are fundamental since they are valid at arbitrary short and long distance scales. Another class of fundamental theories a lá Wilson are the ones featuring an ultraviolet interacting fixed point, and known as asymptotically safe theories. The first proof of existence of asymptotically safe gauge-Yukawa theories in four dimensions appeared in [5]. These type of theories constitute now an important alternative to asymptotic freedom. One can now imagine new extensions of the Standard Model [6-10] and novel ways to achieve radiative symmetry breaking $[6,7]$.

In the original construction [5] elementary scalars and their induced Yukawa interactions play a crucial role in helping make the overall gauge-Yukawa theory safe. Quite surprisingly supersymmetric cousins of the original model, such as super QCD (SQCD) with(out) a meson and Yukawa-like superpotentials, do not support asymptotic safety [11]. An alleged UV fixed point, when asymptotic freedom is lost, would typically violate the $a$-theorem [12-14] inequality [11]. It is possible to go around this constraint, as we shall see in much detail below, by considering theories with multiple fields in distinct matter representations with(out) superpotentials. ${ }^{1}$

Away from perturbation theory supersymmetry allows us to use a plethora of consistency checks, from unitarity to the a-theorem. These tools help constraining the possible

\footnotetext{
${ }^{1}$ We will assume that the list of chiral operators doesn't change for the UV and IR theory. We allow, however, for the $\mathrm{R}$ charges to differ provided all constraints are satisfied. This is similar to what is typically assumed when analysing asymptotically free field theories featuring IR fixed points. Here the same fundamental degrees of freedom can be used to build all the relevant gauge singlet chiral operators in the strongly interacting IR regime and in the perturbative UV limit.
} 
existence of conformal field theories. However the theories passing these tests should still be viewed as potential candidates for a physical fixed point. This is true, of course, also for the celebrated conformal window of SQCD and its generalizations, as well as for all (old and new) candidates for UV fixed points we will present in this paper.

The first candidate for a UV safe theory was SQCD with two adjoints, featuring a large enough number of quark superfields [15] and a superpotential. This mechanism has been recently generalized in [16] for phenomenologically motivated $\mathrm{SO}(10)$ gauge theories with $3 \times 16+10+210+126+\overline{126}[17-19]$ matter representations. The latter is dictated by the requirement that the $R$-parity [20-22] is present at all scales [23-25]. These theories have all $R$-charges uniquely determined because of the presence of the superpotential and the vanishing of the all-order NSVZ beta function [26]. One can consider vanishing superpotentials but then one has to resort to $a$-maximization [27] to determine the $R$-charges. Explicit examples of this type appeared first in [16].

Here we greatly enlarge these families of UV safe supersymmetric candidates, and in the process we gain further insight on how to construct supersymmetric QFTs consistent with nonperturbatively safety. We also investigate quiver theories in which an interacting UV fixed point flows towards an interacting IR one.

The paper is constructed as follows: in section 2 we investigate SQCD with two adjoint fields and different superpotentials. Section 3 contains a study of $\mathrm{SO}(10)$ and $\mathrm{SU}(5)$ gauge theories with different types of vector and chiral like matter without superpotential. Quiver theories are studied in section 4, and we offer our conclusions in section 5.

\section{Safe SQCD with two adjoints and superpotential}

In [15] Martin and Wells proposed a theory for which the nonperturbative existence of an interacting UV fixed point is not excluded by any known constraints. The model features the following superpotential:

$$
W=\operatorname{Tr}[\tilde{Q} X Q]+\operatorname{Tr}\left[X^{3}\right]
$$

and its field content is summarised in table 1. We arrange the number of colours and flavours such that asymptotic freedom is lost and define the quantity $x=N_{c} / N_{f}$. We assume that both terms in the superpotential remain in the UV. ${ }^{2}$ The vanishing of the $\beta$ function for the gauge and holomorphic coupling provides enough constraints to uniquely determine all the $R$-charges of the theory at the would be UV fixed point. Moreover, the anomalous dimensions of the gauge singlet operators do not violate the unitarity bound. The $\Delta a$ between the non trivial fixed point and the IR gaussian turns out to be:

$$
\Delta a=a_{\mathrm{FP}}-a_{\mathrm{FREE}}=\frac{1}{9 x}(1-4 x)(x-1)^{2} .
$$

The non trivial UV fixed point can occur when $x<1 / 4$.

\footnotetext{
${ }^{2}$ The case when one or both of them become irrelevant in the UV can be analyzed by employing $a$ maximization.
} 


\begin{tabular}{|c|c|ccc|}
\hline Fields & {$\left[\mathrm{SU}\left(N_{c}\right)\right]$} & $\mathrm{SU}\left(N_{f}\right)$ & $U_{V}(1)$ & $\mathrm{U}(1)_{R}$ \\
\hline$W_{\alpha}$ & $\operatorname{Adj}$ & 1 & 0 & 1 \\
$Q$ & $\square$ & $\square$ & 1 & $2 / 3$ \\
$\widetilde{Q}$ & $\square$ & $\square$ & -1 & $2 / 3$ \\
$X$ & $\operatorname{Adj}$ & 1 & 0 & $2 / 3$ \\
$Y$ & $\operatorname{Adj}$ & 1 & 0 & $\frac{1}{3}\left(1+\frac{N_{f}}{N_{c}}\right)$ \\
\hline
\end{tabular}

Table 1. The $\mathcal{N}=1$ superfield content with the addition of two gauge adjoint chiral superfield $X, Y$ in the model by Martin and Wells.

It can be shown that this example is part of a larger class of theories defined by the superpotential

$$
W \sim \operatorname{Tr}\left[(\tilde{Q} Q)^{n} X^{k_{1}} Y^{k_{2}}\right]+\operatorname{Tr}\left[(\tilde{Q} Q)^{m} X^{l_{1}} Y^{l_{2}}\right] .
$$

where, as before, we assume that these terms in the superpotential are marginal in the UV for some specific choices of $n, k_{1}, k_{2}, m, l_{1}, l_{2}$. The symbol $\sim$ means that we identify all the superpotentials obtained rearranging the fields in different ways that yield the same $R$ charge constraints. The latter together with the vanishing of the NSVZ beta function gives:

$$
\begin{aligned}
2 n R_{Q}+k_{1} R_{X}+k_{2} R_{Y} & =2 . \\
2 m R_{Q}+l_{1} R_{X}+l_{2} R_{Y} & =2, \\
x\left(R_{X}+R_{Y}-1\right)+\left(R_{Q}-1\right) & =0 .
\end{aligned}
$$

To avoid the emergence of free gauge invariants operators we impose:

$$
n=0 \wedge 2 \leq k_{1}+k_{2} \leq 6 \quad \vee \quad k_{1}=k_{2}=0 \wedge 1 \leq n \leq 3 .
$$

We can find a total of 104 potentials providing UV fixed point satisfying all constraints. Every fixed point satisfies the constraints only in a finite $x$-interval. For example, for $x=0.46$ which is the highest possible value of $x$ allowing an UV interacting fixed point connected to the IR free one, we have seven relevant operators. These potentials read:

$$
\begin{aligned}
& W_{1} \sim \operatorname{Tr}\left[X^{6}\right]+\operatorname{Tr}\left[\tilde{Q} Q X^{4}\right], \\
& W_{2} \sim \operatorname{Tr}\left[X^{6}\right]+\operatorname{Tr}\left[(\tilde{Q} Q)^{2} X^{2}\right], \\
& W_{3} \sim \operatorname{Tr}\left[X^{6}\right]+\operatorname{Tr}\left[(\tilde{Q} Q)^{3}\right], \\
& W_{4} \sim \operatorname{Tr}\left[\tilde{Q} Q X^{4}\right]+\operatorname{Tr}\left[(\tilde{Q} Q)^{2} X^{2}\right], \\
& W_{5} \sim \operatorname{Tr}\left[\tilde{Q} Q X^{4}\right]+\operatorname{Tr}\left[(\tilde{Q} Q)^{3}\right], \\
& W_{6} \sim \operatorname{Tr}\left[(\tilde{Q} Q)^{2} X^{2}\right]+\operatorname{Tr}\left[(\tilde{Q} Q)^{3}\right], \\
& W_{7} \sim \operatorname{Tr}\left[X^{5}\right]+\operatorname{Tr}\left[(\tilde{Q} Q)^{3}\right] .
\end{aligned}
$$

Notice that, at the fixed point, the $R$-charges are the same for the first six potentials implying that the UV value of the $a$-function is the same. This implies that some or all 
of the operators above can be added to the superpotential simultaneously. In addition we expect a manifold of fixed points rather than an isolated one, see for example [28] for such examples in the IR and [16] for UV fixed points. The actual existence of the fixed point manifold, or part of it, needs further evidence that goes beyond passing all known consistency checks. Furthermore the $a$-theorem variation in between any of these UV fixed point and the trivial IR one is positive for small $x$.

\section{Safety without superpotentials: the $\mathrm{SO}(10)$ and $\mathrm{SU}(5)$ templates}

We had already noticed in [16] that all the known bounds for the possible existence of nonperturbative fixed points

$$
\begin{aligned}
\Delta a & >0 \\
c & >0 \\
1 / 6 & \leq(a / c) \leq 1 / 2,
\end{aligned}
$$

are abided with no gauge invariant operators (GIO) with $R<2 / 3$ by, for example, for an $\mathrm{SO}(10)$ theory featuring a very large number of generations respectively in the 10 and 126 representation and with vanishing superpotentials. It is therefore timely to generalise these results.

In the following, the choice of gauge groups $\mathrm{SO}(10)$ or $\mathrm{SU}(5)$ and their representations is partially inspired by the fundamental role they play in grand unified extensions of the Standard Model [29-31]. Supersymmetry is a natural playground for the unification scenario since it almost automatically predicts the correct low energy spectrum that allows for one step-unification of the 3 gauge couplings [32-35]. As discussed in [16], however, asymptotic freedom is never respected in supersymmetric GUTs such as the ones that predict exact R-parity conservation [20-22] at low energy [23-25]. The reason being that one needs large matter representations $[17-19,36]$ under $\mathrm{SO}(10)$, making our current investigation potentially interesting for this line of research.

\subsection{The $\mathrm{SO}(10)$ template}

We start by considering susy $\mathrm{SO}(10)$ theories with $n_{1}$ generations in the representation $r_{1}$ and $n_{2}$ in the representation $r_{2}$ with vanishing superpotential.

We scan for $r_{1}$ and $r_{2}>r_{1}$ over the representations

$$
10,16,45,54,120,126,144,210 \text {. }
$$

The constraint of no GIO with $R<2 / 3$ is satisfied by imposing $R>1 / 3$ for real representations and $R>1 / 6$ for complex representations and we discover that the only solutions satisfying (3.1)-(3.3) above occur for

$$
\left(r_{1}, r_{2}\right)=(10,126),(16,126) .
$$

The number of generations involved is large. The reason being that to abide all the constraints one needs at least $n_{10} \geq 554$, while in the second case $n_{16} \geq 418$. In fact, we now 
argue that there is an infinite number of such solutions for integer number of generations in the 126 representation. To prove this we note that for $n_{10} \geq 8490$ in the first case and for $n_{16} \geq 6191$ in the second case there is at least one integer value of $n_{2}$ for which all constraints (3.1)-(3.3) are satisfied. Since there is no upper bound on $n_{10}$ or $n_{16}$, there is no upper bound on the number of solutions. We now turn our attention to the possibility of having a smaller number of matter fields, but clearly still above the critical number needed to abide the constraints. We find that the most minimal among these solutions contains $n_{1}=478$ generations of 16 and $n_{2}=19$ generations of 126 . For this example we analyze the flow via the Lagrange multiplier technique [37] which for two type of chiral matter reduces to

$$
a=2 G+n_{1} r_{1} a_{1}\left(R_{1}\right)+n_{2} r_{2} a_{1}\left(R_{2}\right)+\lambda_{G}\left(T_{G}+n_{1} T_{1}\left(R_{1}-1\right)+n_{2} T_{2}\left(R_{2}-1\right)\right)
$$

Extremization over $R_{i}, i=1,2$, gives

$$
R_{i}\left(\lambda_{G}\right)=1-\frac{\epsilon_{i}}{3} \sqrt{1-\frac{\lambda_{G} T_{i}}{r_{i}}} \quad, \quad \epsilon_{i}^{2}=1
$$

In the IR $\left(\lambda_{G}=0\right)$ the theory is free, so we are in the $\epsilon_{1,2}=+1$ branch. The flow goes from the IR towards positive $\lambda_{G}$ (that it must be positive here we know from perturbative calculations which are applicable for small enough $\lambda_{G} \sim g^{2}$ ) until it reaches

$$
\lambda_{G}^{\max } \equiv \operatorname{Min}\left(r_{i} / T_{i}\right)
$$

which is, in the two cases (3.5), always given by 126 :

$$
\lambda_{G}^{\max }=\frac{126}{35}=\frac{18}{5}=3.6
$$

At this point $\epsilon_{126}$ changes sign. $\lambda_{G}$ can now only decrease (increasing above $\lambda_{G}^{\max }$ would lead to complex value for $R_{126}$ ), but now in the branch $\epsilon_{16}=+1, \epsilon_{126}=-1$. We pass through $\lambda_{G}=0$ (which is no more a free theory, because of the different branch) towards negative values of $\lambda_{G}$, all the way to the fixed point value of

$$
\lambda_{G}^{*}=-41.63
$$

for which (having $a(0)=20894 / 9)$ :

$$
\begin{aligned}
\left(R_{16}, R_{126}\right)\left(\lambda_{G}^{*}\right) & =(0.1697,2.1816) \\
a\left(\lambda_{G}^{*}\right) & =2326.5 \\
\Delta a\left(\lambda_{G}^{*}\right) & =4.955 \\
c\left(\lambda_{G}^{*}\right) & =13932.1 \\
(a / c)\left(\lambda_{G}^{*}\right)-1 / 6 & =3.2 \times 10^{-4}
\end{aligned}
$$

Notice that $4 R_{16}>2 / 3$ but in order to avoid a free field with $R=2 R_{16}<2 / 3$ we need to have only 16 or only $\overline{16}$ but not both (i.e. we cannot have $n_{1} / 2=239$ copies of 16 and 239 copies of $\overline{16}$ ). This is in principle not necessary for the 126 for which the $R_{126}$ is 


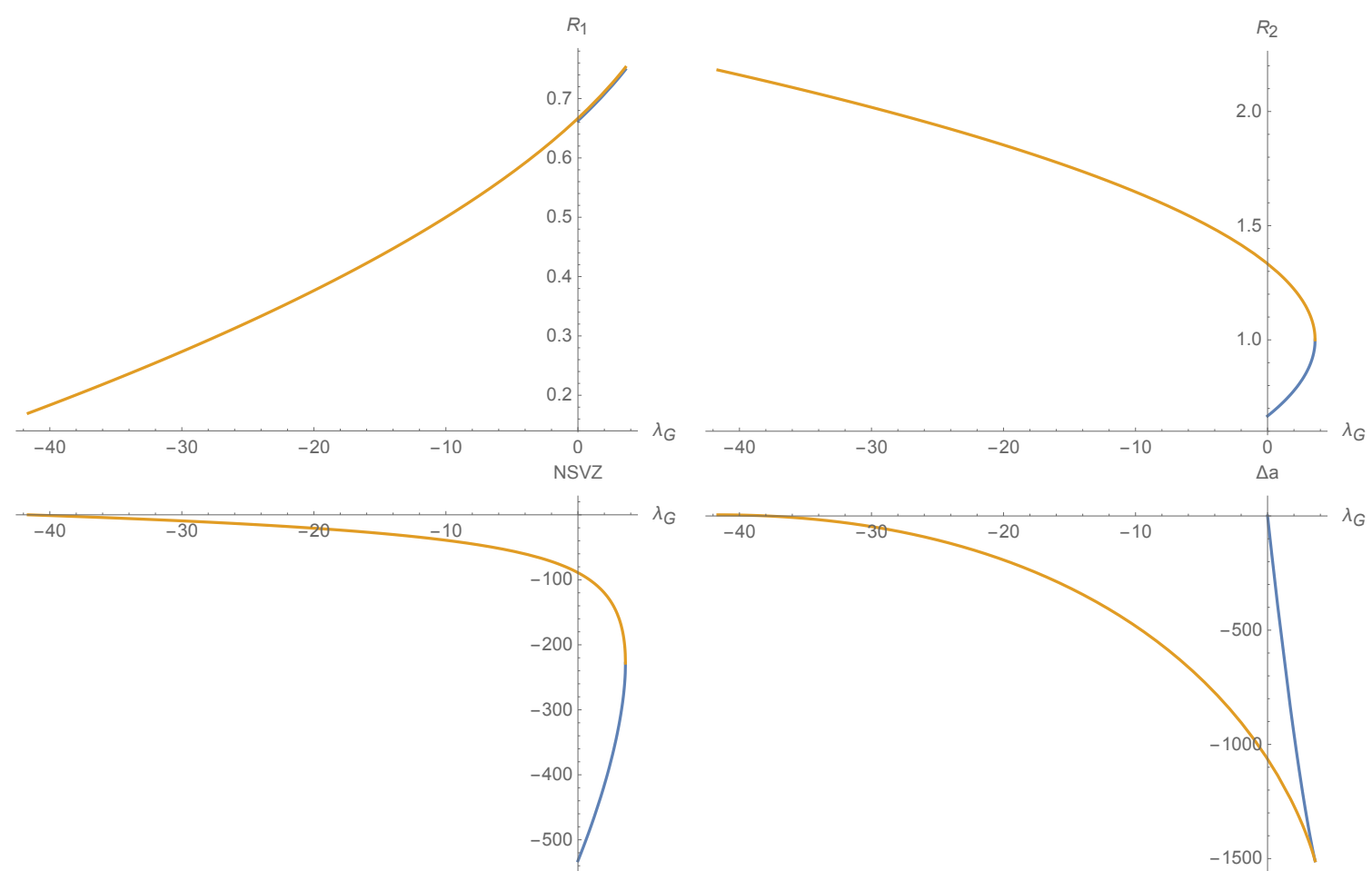

Figure 1. Flows of $R_{1}$ (upper left), $R_{2}$ (upper right), the $N S V Z$ function (lower left) and $\Delta a$ (lower right) as functions of the Lagrange multiplier $\lambda_{G}$. The blue (orange) curves show the direction of the flow towards increasing (decreasing) $\lambda_{G}$. The starting points of the blue curves at $\lambda_{G}=0$ are at the free IR theory.

safely large, but since their total number $n_{2}=19$ is odd, we cannot have half of them in representation 126 and half of them in $\overline{126}$ to maintain the exchange symmetry between them (see also the comment in the following section). The flows of the different quantities are shown in figure 1.

We have therefore found an entire family of solutions that can be asymptotically safe. Furthermore, the fact that there is a critical number of matter field value above which the asymptotically safe theory emerges within infrared gauge-matter free theories can be viewed as the supersymmetric analogue of the large $N_{f}$ solutions of non-supersymmetric safe non-abelian gauge-fermion theories recently discussed in [38].

\subsection{The SU(5) template}

One can repeat the above analysis for $\mathrm{SU}(5)$. Considering only fields up to representation 75 , i.e. over

$$
5,10,15,24,35,40,45,50,70,70^{\prime}, 75
$$

(and their conjugates) only the following pairs can lead to consistent UV fixed point and free IR limits (to be on the safe side we impose here $R_{i} \geq 1 / 3$ for all, real or complex representations; also, we assume that the $R$ charges of a field in representation $r$ is the 


\begin{tabular}{|c|c|c|c|c|c|c|c|c|}
\hline$r_{1}$ & $n_{1}+n_{\overline{1}}$ & $R_{1}=R_{\overline{1}}$ & $r_{2}$ & $n_{2}+n_{\overline{2}}$ & $R_{2}=R_{\overline{2}}$ & $\Delta a$ & $c_{U V}$ & $(a / c)_{U V}$ \\
\hline 5 & 180 & 0.36651 & 70 & 2 & 2.06152 & 6.38 & 1652. & 0.173 \\
5 & 180 & 0.35869 & 75 & 2 & 2.05436 & 0.99 & 1637. & 0.172 \\
10 & 102 & 0.43853 & 70 & 2 & 1.96316 & 13.7 & 1786. & 0.179 \\
\hline
\end{tabular}

Table 2. The candidates for UV fixed points with the minimal number of generations in the SU(5) example.

same as the $R$ charge of an eventual field in the conjugate representation $\bar{r}$ ):

$$
\left(r_{1}, r_{2}\right)=(5,35),(5,40),(5,70),\left(5,70^{\prime}\right),(5,75),\left(10,70^{\prime}\right),\left(24,70^{\prime}\right)
$$

Differently from $\mathrm{SO}(10)$, these $\mathrm{SU}(5)$ examples are not automatically anomaly free. In chiral theories the number of fields $n_{1,2}$ is different from the number of antifields $n_{\overline{1}, \overline{2}}$, and so the $R$-charges of fields and antifields is, in general, different. ${ }^{3}$ In this case one should maximise the $a$ function over 3 different $R$-charges, i.e. $R_{1,2, \overline{1}, \overline{2}}$ subject to the vanishing of the NSVZ beta function. We therefore restrict the analysis over vectorlike examples, e.g. $n_{\overline{1}}=n_{1}$ and $n_{\overline{2}}=n_{2}$. In this way the system is symmetric under the exchange of fields with antifields, and only one independent $R$ charge remains after imposing the vanishing of the NSVZ beta function. There is an infinite number of solutions, let's present those with the minimal number of generations, taking into account only solutions with

$$
n_{1} \leq 500 \quad, \quad n_{2} \leq 2
$$

We summarise them in table 2. Notice that in order to have the precision in $\Delta a$ as specified in table 2 we had to specify $R_{1,2}$ with higher precision, since cancellations are at work.

These new families of solutions show that supersymmetric gauge theories with(out) chiral matter and without superpotential can be asymptotically safe above a critical number of matter fields. Our results complement the investigation for non supersymmetric chiral gauge theories performed first in [39]. Our analysis can be straightforwardly extended to other gauge groups with similar matter content. One can also relax the constraints on the absence of GIO operators but this will be explored elsewhere.

\section{Semi-simple gauge groups}

Here we will analyse examples of semi-simple gauge groups starting with the quivers of $[27,40]$.

\subsection{The $\mathrm{SU}(\mathrm{N})^{4}$ quiver}

The field content of the theory along with the gauge and $\mathrm{SU}(2)$ flavor symmetries and charges are shown in table 3 . In the $N \rightarrow \infty$ limit one recovers the $\mathrm{U}(\mathrm{N})^{4}$ case. We

\footnotetext{
${ }^{3}$ We thank the referee for helping correcting a statement in the first version of this work.
} 


\begin{tabular}{|c|ccccc|}
\hline Fields & {$\left[\mathrm{SU}(N)_{1}\right]\left[\mathrm{SU}(N)_{2}\right]\left[\mathrm{SU}(N)_{3}\right]\left[\mathrm{SU}(N)_{4}\right]$} & $\mathrm{SU}(2)_{F}$ \\
\hline$X_{21}^{a}$ & $\overline{1}$ & $\square$ & 1 & 1 & $\square$ \\
$X_{21}^{3}$ & $\square$ & $\square$ & 1 & 1 & 1 \\
$X_{14}^{a}$ & $\square$ & 1 & 1 & $\square$ & $\square$ \\
$X_{43}$ & 1 & 1 & $\overline{1}$ & $\square$ & 1 \\
$X_{32}^{a}$ & 1 & $\square$ & $\square$ & 1 & $\square$ \\
$X_{13}$ & $\square$ & 1 & $\square$ & 1 & 1 \\
$X_{42}$ & 1 & $\square$ & 1 & $\square$ & 1 \\
\hline
\end{tabular}

Table 3. The quantum numbers of the field content in the quiver example.

consider a superpotential that respects all the symmetries:

$$
\begin{aligned}
W & =y_{1} \mathcal{O}_{1}+y_{2} \mathcal{O}_{2}+y_{3} \mathcal{O}_{3} \\
& =\operatorname{Tr}\left[y_{1} \epsilon_{a b} X_{21}^{a} X_{14}^{b} X_{42}+y_{2} \epsilon_{a b} X_{21}^{a} X_{13} X_{32}^{b}+y_{3} \epsilon_{a b} X_{21}^{3} X_{14}^{a} X_{43} X_{32}^{b}\right] .
\end{aligned}
$$

We study the following cases:

1. if $y_{3}=0$ there is a free field solution, with all $R_{i}=2 / 3$ and

$$
\begin{aligned}
\frac{a}{N^{2}} & =\frac{92}{9}-\frac{8}{N^{2}} \stackrel{N \rightarrow \infty}{\longrightarrow} 10.2 \\
\frac{a}{c} & =\frac{23-18 / N^{2}}{66-36 / N^{2}} \stackrel{N \rightarrow \infty}{\longrightarrow} 0.348
\end{aligned}
$$

2. if $y_{1}=y_{2}=y_{3}=0$ (i.e. $W=0$ ) we have [27]

$$
\begin{aligned}
\frac{a}{N^{2}} & =\frac{2}{3}(3+5 \sqrt{5})-\frac{8}{N^{2}} \stackrel{N \rightarrow \infty}{\longrightarrow} 9.45 \\
\frac{a}{c} & =\frac{\frac{2}{3}(3+5 \sqrt{5})-\frac{8}{N^{2}}}{2(3+5 \sqrt{5})-\frac{16}{N^{2}}} \stackrel{N \rightarrow \infty}{\longrightarrow} \frac{1}{3}
\end{aligned}
$$

The $R$-charges of the operators defined in (4.1) are independent on $N$ and equal to

$$
R\left(\mathcal{O}_{1}\right)=1.87 \quad, \quad R\left(\mathcal{O}_{2}\right)=1.87 \quad, \quad R\left(\mathcal{O}_{3}\right)=2.25
$$

3. finally, if any of the $y_{i} \neq 0$ (i.e. if $W \neq 0$ ), we get [40]

$$
\begin{aligned}
\frac{a}{N^{2}} & =\frac{32}{3}(-46+13 \sqrt{13})-\frac{8}{N^{2}} \stackrel{N \rightarrow \infty}{\longrightarrow} 9.30 \\
\frac{a}{c} & =\frac{\frac{32}{3}(-46+13 \sqrt{13})-\frac{8}{N^{2}}}{32(-46+13 \sqrt{13})-\frac{16}{N^{2}}} \stackrel{N \rightarrow \infty}{\longrightarrow} \frac{1}{3}
\end{aligned}
$$

The $R$-charges of the operators defined in (4.1) are

$$
R\left(\mathcal{O}_{1}\right)=2 \quad, \quad R\left(\mathcal{O}_{2}\right)=2 \quad, \quad R\left(\mathcal{O}_{3}\right)=2
$$




\begin{tabular}{|c|cccc|}
\hline Fields & {$\left[\mathrm{SU}\left(N_{1}\right)\right]$} & $\left.\mathrm{SU}\left(N_{2}\right)\right]$ & $\mathrm{SU}\left(N_{f}\right)$ & $\mathrm{SU}\left(N_{q}\right)$ \\
\hline$\psi$ & $\square$ & 1 & $\bar{\square}$ & 1 \\
$\widetilde{\psi}$ & $\square$ & 1 & $\square$ & 1 \\
$\Psi$ & $\square$ & $\square$ & 1 & 1 \\
$\widetilde{\Psi}$ & $\square$ & $\square$ & 1 & 1 \\
$\widetilde{\chi}$ & 1 & $\square$ & $\square$ & 1 \\
$\chi$ & 1 & $\square$ & $\bar{\square}$ & 1 \\
$\widetilde{Q}$ & 1 & $\square$ & 1 & $\square$ \\
$Q$ & 1 & $\square$ & 1 & $\square$ \\
\hline
\end{tabular}

Table 4. Field content of the model introduced in [44].

In the limit $N \rightarrow \infty$, the ratio $a / c$ approaches $1 / 3$ for the case of point 2 and 3 , in agreement with the large $N$ expectation for superconformal quivers [41, 42]. ${ }^{4}$

Requiring

(a) $\Delta a \equiv a_{U V}-a_{I R}>0$

(b) if $y_{i} \neq 0$ then $R\left(\mathcal{O}_{i}\right) \geq 2$ in the IR and $R\left(\mathcal{O}_{i}\right) \leq 2$ in the UV,

we find that the only UV safe flow is for a vanishing superpotential $W=0$ in the UV (case 2) and for an interacting $W \neq 0$ but with $y_{3}=0$ in the IR (case 3 ); in this scenario we have both an IR and an UV interacting fixed point candidates.

\subsection{An $\mathrm{SU}\left(\mathrm{N}_{1}\right) \otimes \mathrm{SU}\left(\mathrm{N}_{2}\right)$ example}

Theories with safe trajectories for semisimple gauge groups were first analysed and discovered in [43]. For these theories it is possible to achieve RG trajectories connecting UV and IR interacting fixed points. A supersymmetric model of this type was considered in [44] which can be also viewed as a variant of the $\mathrm{SU}(N)^{4}$ quiver in which one gauges two of the previous non-abelian flavour symmetries. We summarise in table 4 the field content.

The model features in addition a Yukawa-type superpotential of the form:

$$
W=y(\operatorname{Tr}[\psi \widetilde{\Psi} \chi]+\operatorname{Tr}[\widetilde{\psi} \Psi \widetilde{\chi}])
$$

We will consider the model in the Veneziano limit keeping the following ratios fixed:

$$
x_{1}=\frac{N_{1}}{N_{f}}, \quad x_{2}=\frac{N_{2}}{N_{f}}, \quad x_{q}=\frac{N_{q}}{N_{f}} .
$$

\footnotetext{
${ }^{4}$ The reason is [42], that in this limit the $\operatorname{Tr} \mathrm{U}(1)_{R}$ is proportional to the weighted sum of the NSVZ $\beta$ functions, and thus zero at a superconformal fixed point. Since by definition the same trace is proportional to $a-(c / 3)$, the relation $a / c=1 / 3$ follows automatically for any quiver superconformal gauge theory.
} 
The $\beta$ function for the gauge and superpotential couplings are:

$$
\begin{aligned}
& \beta_{y}=\frac{3}{2} y\left(R_{\psi}+R_{\Psi}+R_{\chi}-2\right) \\
& \beta_{1}=-\frac{3 g_{1}^{3}}{16 \pi^{2}} f\left(g_{1}^{2}\right)\left[x_{1}+\left(R_{\psi}-1\right)-x_{2}\left(R_{\Psi}-1\right)\right] \\
& \beta_{2}=-\frac{3 g_{2}^{3}}{16 \pi^{2}} f\left(g_{2}^{2}\right)\left[x_{2}-\left(R_{\chi}-1\right)+x_{1}\left(R_{\Psi}-1\right)+x_{q}\left(R_{Q}-1\right)\right]
\end{aligned}
$$

where $f\left(g^{2}\right) \sim 1+O\left(g^{2}\right)$ is a scheme dependent function of the couplings. The properly normalized $a$-function reads:

$$
a / N_{f}^{2}=2\left(x_{1}^{2}+x_{2}^{2}\right)+2 x_{1} a_{1}\left(R_{\psi}\right)+2 x_{2} a_{1}\left(R_{\chi}\right)+2 x_{1} x_{2} a_{1}\left(R_{\Psi}\right)+2 x_{q} x_{2} a_{1}\left(R_{Q}\right) .
$$

We can now find the nonperturbative candidate fixed points of the theory by setting to zero the beta functions together with a-maximisation. We also allow for partially interacting fixed points, following [43], meaning that some of the beta functions vanish trivially at the origin of their respective couplings. To compare our nonperturbative results with the perturbative ones given in [44] we introduce the further quantities:

$$
P_{1}=\frac{x_{2}}{x_{1}}, \quad P_{2}=\frac{x_{2}}{x_{1}} \frac{x_{q}+x_{1}-3 x_{2}+1}{x_{2}-3 x_{1}+1}, \quad \epsilon=\frac{x_{2}-3 x_{1}+1}{x_{1}},
$$

and assume $P_{1}=3 / 2$ and $P_{2}=-5$ while, differently from [44], our $\epsilon$ can take any positive value in the range $] 0,0.16776$ ] for which no free GIO can emerge. We find seven distinct potential fixed points including the fully non-interacting one in all couplings that pass all the known nonperturbative tests. Of these fixed points three are the physical ones that go over the perturbative analysis. Ordering in the descending value assumed by the central charge $a$ these are the gaussian fixed point at the origin $G$ of all couplings, the interacting $\left(\mathrm{FP}_{2 y}\right)$ in all couplings except $\alpha_{1}$ and the fully interacting one $\left(\mathrm{FP}_{12 y}\right)$. We report in figure 2 the nonperturbative $R$ charges for the (semi)interacting fixed points as functions of $\epsilon$.

With these charges we plot in figure 3 the value of $a$ and $a / c$ as functions of $\epsilon$.

It is clear from the figure that all bounds are respected and that furthermore the highest value of $a$ is for $\mathrm{FP}_{2 y}$ suggesting that if a flow exists between this and the fully interacting fixed point, it can be seen as an ultraviolet safe fixed point along this trajectory. This is the susy equivalent of the phenomenon discovered in [43]. In addition we also notice that the fully gaussian fixed point has the highest possible value of $a$ establishing an hierarchy of UV fixed points according to which, de facto, any phenomenological interesting field theory of this type would eventually flow to the fully gaussian one. This is substantially different from the case of [5] in which, at least perturbatively, the only UV fixed point has the maximum $a$. In addition we expect no separatrix directly connecting $\mathrm{FP}_{12 y}$ with the gaussian fixed point but a separatrix along the $\alpha_{2}$ coupling direction connecting it to $\mathrm{FP}_{2 y}$ because the linearised flow around the gaussian fixed point must necessarely coincide with the perturbative analysis. 

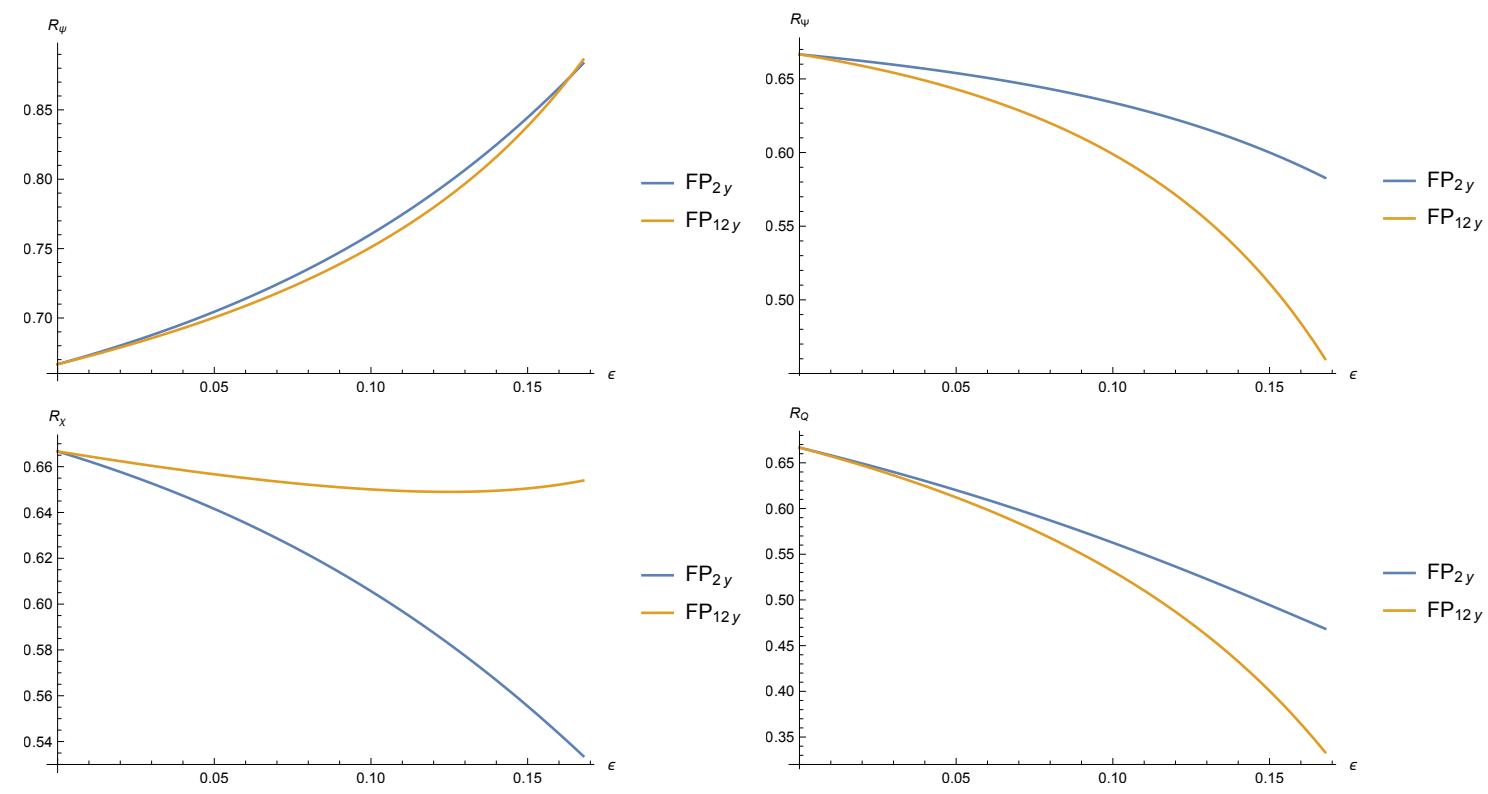

Figure 2. Here we draw the $R$-charges as function of the parameter $\epsilon$ for fixed $P_{1}=3 / 2$ and $P_{2}=-5$.
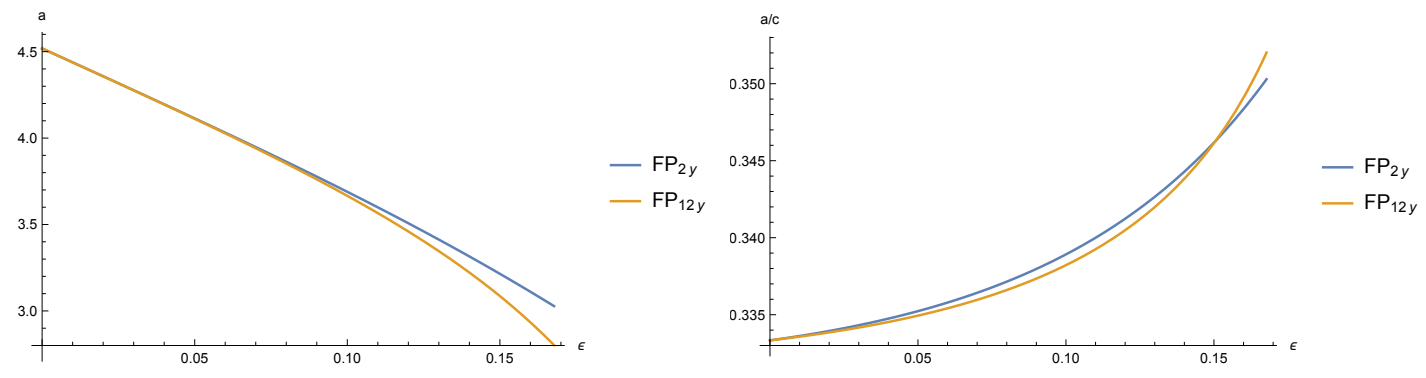

Figure 3. $a$ and $a / c$ as a functions of $\epsilon$ for the (semi)interacting fixed points.

\section{Conclusions}

We studied the short distance behaviour of several distinct classes of not asymptotically free supersymmetric gauge theories. In particular we investigated super QCD with two adjoint fields and generalised superpotentials. Here we showed that candidates for nonperturbative asymptotic safety can be achieved without violating the known constraints provided the superpotentials assume specific forms.

We also investigated the possible emergence of asymptotic safety within supersymmetric field theories featuring only gauge interactions. We discovered that asymptotic safety could be achieved at the cost of introducing a large enough number of matter fields in distinct representations of the gauge groups. In addition we investigated also semi-simple gauge theories with superpotentials such as quiver theories, and demonstrated that asymptotic safety could be achieved as well. Here the mechanism at play requires connecting the UV safe theory to an interacting IR one. 
Our results integrate and extend the initial work of ref. [11] by introducing new mechanisms to achieve supersymmetric safety.

\section{Acknowledgments}

BB acknowledges the financial support from the Slovenian Research Agency (research core funding No. P1-0035). The work of ND and FS is partially supported by the Danish National Research Foundation under the grant DNRF:90. BB thanks the CERN theoretical physics division for the hospitality.

Open Access. This article is distributed under the terms of the Creative Commons Attribution License (CC-BY 4.0), which permits any use, distribution and reproduction in any medium, provided the original author(s) and source are credited.

\section{References}

[1] D.J. Gross and F. Wilczek, Asymptotically Free Gauge Theories. 1, Phys. Rev. D 8 (1973) 3633 [INSPIRE].

[2] H.D. Politzer, Reliable Perturbative Results for Strong Interactions?, Phys. Rev. Lett. 30 (1973) 1346 [INSPIRE].

[3] K.G. Wilson, Renormalization group and critical phenomena. 1. Renormalization group and the Kadanoff scaling picture, Phys. Rev. B 4 (1971) 3174 [INSPIRE].

[4] K.G. Wilson, Renormalization group and critical phenomena. 2. Phase space cell analysis of critical behavior, Phys. Rev. B 4 (1971) 3184 [INSPIRE].

[5] D.F. Litim and F. Sannino, Asymptotic safety guaranteed, JHEP 12 (2014) 178 [arXiv: 1406.2337] [INSPIRE].

[6] S. Abel and F. Sannino, Radiative symmetry breaking from interacting UV fixed points, Phys. Rev. D 96 (2017) 056028 [arXiv:1704.00700] [INSPIRE].

[7] S. Abel and F. Sannino, Framework for an asymptotically safe Standard Model via dynamical breaking, Phys. Rev. D 96 (2017) 055021 [arXiv:1707.06638] [INSPIRE].

[8] G.M. Pelaggi, F. Sannino, A. Strumia and E. Vigiani, Naturalness of asymptotically safe Higgs, Front. in Phys. 5 (2017) 49 [arXiv:1701.01453] [INSPIRE].

[9] R. Mann, J. Meffe, F. Sannino, T. Steele, Z.-W. Wang and C. Zhang, Asymptotically Safe Standard Model via Vectorlike Fermions, Phys. Rev. Lett. 119 (2017) 261802 [arXiv: 1707.02942] [INSPIRE].

[10] G.M. Pelaggi, A.D. Plascencia, A. Salvio, F. Sannino, J. Smirnov and A. Strumia, Asymptotically Safe Standard Model Extensions, arXiv:1708.00437 [INSPIRE].

[11] K. Intriligator and F. Sannino, Supersymmetric asymptotic safety is not guaranteed, JHEP 11 (2015) 023 [arXiv: 1508.07411] [INSPIRE].

[12] J.L. Cardy, Is There a c Theorem in Four-Dimensions?, Phys. Lett. B 215 (1988) 749 [INSPIRE].

[13] Z. Komargodski and A. Schwimmer, On Renormalization Group Flows in Four Dimensions, JHEP 12 (2011) 099 [arXiv: 1107.3987] [InSPIRE]. 
[14] Z. Komargodski, The Constraints of Conformal Symmetry on RG Flows, JHEP 07 (2012) 069 [arXiv: 1112.4538] [INSPIRE].

[15] S.P. Martin and J.D. Wells, Constraints on ultraviolet stable fixed points in supersymmetric gauge theories, Phys. Rev. D 64 (2001) 036010 [hep-ph/0011382] [INSPIRE].

[16] B. Bajc and F. Sannino, Asymptotically Safe Grand Unification, JHEP 12 (2016) 141 [arXiv: 1610.09681] [INSPIRE].

[17] T.E. Clark, T.-K. Kuo and N. Nakagawa, A SO(10) Supersymmetric Grand Unified Theory, Phys. Lett. B 115 (1982) 26 [inSPIRE].

[18] C.S. Aulakh and R.N. Mohapatra, Implications of Supersymmetric $\mathrm{SO}(10)$ Grand Unification, Phys. Rev. D 28 (1983) 217 [INSPIRE].

[19] C.S. Aulakh, B. Bajc, A. Melfo, G. Senjanović and F. Vissani, The minimal supersymmetric grand unified theory, Phys. Lett. B 588 (2004) 196 [hep-ph/0306242] [INSPIRE].

[20] R.N. Mohapatra, New Contributions to Neutrinoless Double beta Decay in Supersymmetric Theories, Phys. Rev. D 34 (1986) 3457 [InSPIRE].

[21] A. Font, L.E. Ibáñez and F. Quevedo, Does Proton Stability Imply the Existence of an Extra Z0?, Phys. Lett. B 228 (1989) 79 [inSPIRE].

[22] S.P. Martin, Some simple criteria for gauged R-parity, Phys. Rev. D 46 (1992) R2769 [hep-ph/9207218] [INSPIRE].

[23] C.S. Aulakh, K. Benakli and G. Senjanović, Reconciling supersymmetry and left-right symmetry, Phys. Rev. Lett. 79 (1997) 2188 [hep-ph/9703434] [INSPIRE].

[24] C.S. Aulakh, A. Melfo and G. Senjanović, Minimal supersymmetric left-right model, Phys. Rev. D 57 (1998) 4174 [hep-ph/9707256] [INSPIRE].

[25] C.S. Aulakh, A. Melfo, A. Rasin and G. Senjanović, Seesaw and supersymmetry or exact R-parity, Phys. Lett. B 459 (1999) 557 [hep-ph/9902409] [INSPIRE].

[26] V.A. Novikov, M.A. Shifman, A.I. Vainshtein and V.I. Zakharov, Exact Gell-Mann-Low Function of Supersymmetric Yang-Mills Theories from Instanton Calculus, Nucl. Phys. B 229 (1983) 381 [INSPIRE].

[27] K.A. Intriligator and B. Wecht, The Exact superconformal R symmetry maximizes a, Nucl. Phys. B 667 (2003) 183 [hep-th/0304128] [INSPIRE].

[28] R.G. Leigh and M.J. Strassler, Exactly marginal operators and duality in four-dimensional $N=1$ supersymmetric gauge theory, Nucl. Phys. B 447 (1995) 95 [hep-th/9503121] [INSPIRE].

[29] J.C. Pati and A. Salam, Lepton Number as the Fourth Color, Phys. Rev. D 10 (1974) 275 [Erratum ibid. D 11 (1975) 703] [INSPIRE].

[30] H. Georgi and S.L. Glashow, Unity of All Elementary Particle Forces, Phys. Rev. Lett. 32 (1974) 438 [INSPIRE].

[31] H. Georgi, H.R. Quinn and S. Weinberg, Hierarchy of Interactions in Unified Gauge Theories, Phys. Rev. Lett. 33 (1974) 451 [InSPIRE].

[32] S. Dimopoulos, S. Raby and F. Wilczek, Supersymmetry and the Scale of Unification, Phys. Rev. D 24 (1981) 1681 [INSPIRE]. 
[33] L.E. Ibáñez and G.G. Ross, Low-Energy Predictions in Supersymmetric Grand Unified Theories, Phys. Lett. B 105 (1981) 439 [INSPIRE].

[34] M.B. Einhorn and D.R.T. Jones, The Weak Mixing Angle and Unification Mass in Supersymmetric SU(5), Nucl. Phys. B 196 (1982) 475 [inSPIRE].

[35] W.J. Marciano and G. Senjanović, Predictions of Supersymmetric Grand Unified Theories, Phys. Rev. D 25 (1982) 3092 [INSPIRE].

[36] K.S. Babu and R.N. Mohapatra, Predictive neutrino spectrum in minimal SO(10) grand unification, Phys. Rev. Lett. 70 (1993) 2845 [hep-ph/9209215] [INSPIRE].

[37] D. Kutasov, New results on the 'a theorem' in four-dimensional supersymmetric field theory, hep-th/0312098 [INSPIRE].

[38] O. Antipin and F. Sannino, Conformal Window 2.0: The Large- $N_{f}$ Safe Story, arXiv: 1709.02354 [INSPIRE].

[39] E. Mølgaard and F. Sannino, Asymptotically safe and free chiral theories with and without scalars, Phys. Rev. D 96 (2017) 056004 [arXiv: 1610.03130] [InSPIRE].

[40] M. Bertolini, F. Bigazzi and A.L. Cotrone, New checks and subtleties for AdS/CFT and a-maximization, JHEP 12 (2004) 024 [hep-th/0411249] [INSPIRE].

[41] M. Henningson and K. Skenderis, The Holographic Weyl anomaly, JHEP 07 (1998) 023 [hep-th/9806087] [INSPIRE].

[42] S. Benvenuti and A. Hanany, New results on superconformal quivers, JHEP 04 (2006) 032 [hep-th/0411262] [INSPIRE].

[43] J.K. Esbensen, T.A. Ryttov and F. Sannino, Quantum critical behavior of semisimple gauge theories, Phys. Rev. D 93 (2016) 045009 [arXiv:1512.04402] [InSPIRE].

[44] A.D. Bond and D.F. Litim, Asymptotic safety guaranteed in supersymmetry, Phys. Rev. Lett. 119 (2017) 211601 [arXiv:1709.06953] [INSPIRE]. 